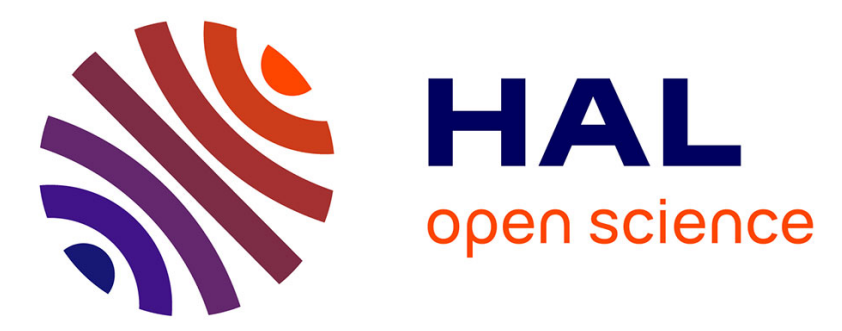

\title{
Bases physiques et performances des nouveaux (micro)capteurs magnétiques à semiconducteur
}

A. Chovet, S. Cristoloveanu

\section{To cite this version:}

A. Chovet, S. Cristoloveanu. Bases physiques et performances des nouveaux (micro)capteurs magnétiques à semiconducteur. Revue de Physique Appliquée, 1984, 19 (2), pp.69-76. 10.1051/rphysap:0198400190206900 . jpa-00245175

\section{HAL Id: jpa-00245175 https://hal.science/jpa-00245175}

Submitted on 1 Jan 1984

HAL is a multi-disciplinary open access archive for the deposit and dissemination of scientific research documents, whether they are published or not. The documents may come from teaching and research institutions in France or abroad, or from public or private research centers.
L'archive ouverte pluridisciplinaire HAL, est destinée au dépôt et à la diffusion de documents scientifiques de niveau recherche, publiés ou non, émanant des établissements d'enseignement et de recherche français ou étrangers, des laboratoires publics ou privés. 
Classification

Physics Abstracts

$72.20 \mathrm{M}-73.60 \mathrm{~F}$

\title{
Bases physiques et performances des nouveaux (micro)capteurs magnétiques à semiconducteur
}

\author{
A. Chovet et S. Cristoloveanu \\ Laboratoire de Physique des Composants à Semiconducteur $\left({ }^{*}\right)$ \\ ENSERG, 23, rue des Martyrs, 38031 Grenoble Cedex, France \\ (Reçu le 3 juin 1983, révisé le 15 septembre, accepté le 19 octobre 1983)
}

\begin{abstract}
Résumé. - Nous présentons ici une synthèse sur les capteurs magnétiques à semiconducteur développés dans notre laboratoire et utilisant l'effet de concentration des porteurs par un champ magnétique. Après avoir rappelé les principes physiques des effets de magnétoconcentration ou magnétodiode, nous donnons les derniers résultats relatifs à l'optimisation des performances. L'accent est mis davantage sur les aspects pratiques que sur des développements à caractère théorique.

Nous donnons aussi les principales caractéristiques des capteurs réalisés sur divers matériaux et. en particulier, celles concernant les micromagnétodiodes intégrées avec la technologie silicium sur corindon. Nous dégageons les paramètres importants pour un fonctionnement optimal (sensibilité, bruit). dans une large gamme de température.

Abstract. - A synthesis on semiconductor magnetic sensors based on the concentration of charge carriers by a magnetic field is presented. Physical principles of magnetoconcentration and magnetodiode effects are reminded before giving last results on device improvement.

The main features of sensors made from different semiconductors are given with a special attention to integrated micromagnetodiodes made with silicon on sapphire technology. The important parameters for the best working (sensitivity, noise) in a wide range of temperature are displayed.
\end{abstract}

\section{Introduction.}

Les capteurs magnétosensibles à semiconducteur traditionnels sont basés sur l'effet Hall (fonctionnement en quadripôle) ou sur des effets de magnétorésistance (fonctionnement en dipôle) dans des matériaux où la mobilité des porteurs de charge électrique est élevée (souvent l'InSb) [1, 2]. Malgré leur grande diffusion et certains avantages, comme la bonne linéarité de la réponse d'une cellule Hall, ces capteurs présentent plusieurs inconvénients : leurs dimensions restent importantes (surface supérieure au $\mathrm{mm}^{2}$ ) et, pour les dispositifs sur InSb, leur réalisation selon une technologie "circuits intégrés" est impossible à l'heure actuelle; de plus, pour l'effet Hall, l'utilisation en quadripôle n'est pas toujours un avantage et, pour l'effet de magnétorésistance, on ne peut détecter la direction du champ magnétique et la sensibilité à bas champ est faible.

$\left.{ }^{*}\right)$ ERA CNRS No 659.
Depuis plusieurs années, nous avons étudié et mis au point divers capteurs de grande sensibilité, intégrables en technologie silicium VLSI et permettant donc d'envisager le développement prochain de capteurs magnétiques " intelligents ", c'est-à-dire associés sur la même "puce" à des circuits de traitement et de décision. Ces capteurs sont basés sur l'effet de concentration de porteurs par un champ magnétique au voisinage d'une des surfaces du dispositif.

Nous présentons ici l'effet de magnétoconcentration et son optimisation pour la réalisation de capteurs magnétiques, puis son extension au cas des magnétodiodes, initialement étudiées en URSS [11, 23, 24] et au Japon par la firme Sony [25], et pour lesquelles les technologies LSI et VLSI ont permis de développer plusieurs types de micro-capteurs intégrés, aussi testés en Allemagne (Siemens) [26], aux USA (Honeywell) [27] et en Suisse ((Landis et Gyr) [28]). Nous donnerons enfin les performances et les limites ultimes (tenue en température, bruit) que nous avons obtenues avec cette famille de capteurs.

Signalons qu'il existe d'autres composants magné- 
tosensibles à semiconducteur réalisables par des technologies « planar » usuelles mais basés sur d'autres principes (tels les magnétotransistors $\operatorname{MOS}[29,30]$ ou bipolaires, en fonctionnement normal [31-33] ou en régime d'avalanche [34,35], ainsi que les dispositifs à résistance négative [36]), qui ne seront pas analysés ici.

\section{L'effet de magnétoconcentration.}

L'effet de magnétoconcentration (MC) [3-5] se manifeste dans les semiconducteurs à comportement ambipolaire (matériaux intrinsèques, quasi-intrinsèques, ou extrinsèques mais tels que $\left.n\left|\mu_{n}\right| \sim p \mu_{p}[6]\right)$.

2.1 PRINCIPE PHYSIQUe. - Considérons un échantillon parallélépipédique d'un tel semiconducteur (initialement isotrope), muni de contacts ohmiques, et auquel on applique des champs électrique $\mathbf{E}_{\boldsymbol{x}}$ et magnétique $\mathbf{B}=\mathbf{B}_{z}$ orthogonaux, d'intensité quelconque (configuration identique à celle d'une expérience de Hall, voir Fig. 1). Nous négligerons ici tout effet lié aux porteurs chauds ou à une magnétorésistance.

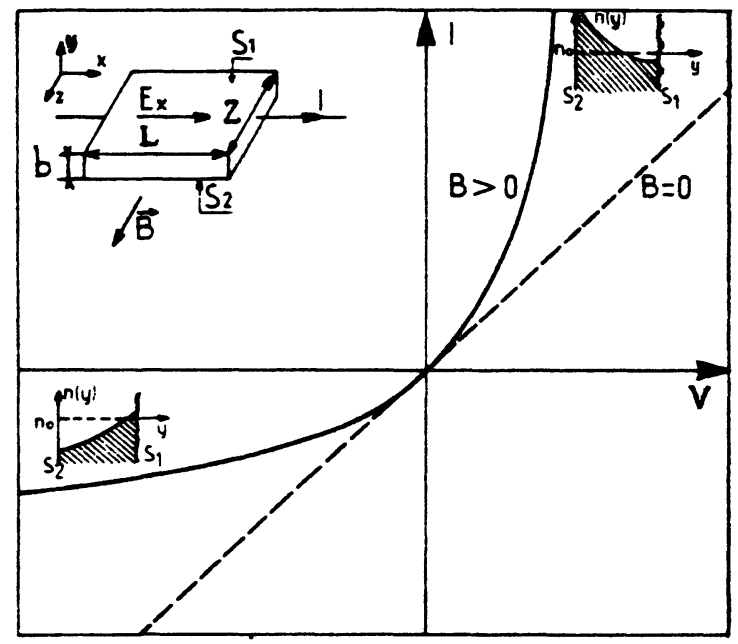

Fig. 1. - Configuration de l'effet de magnétoconcentration (ainsi que de l'effet magnétodiode). Allure des distributions transversales de porteurs $n(y)$ et caractéristique $I(V)$ pour $s_{1} \rightarrow \infty$ et $s_{2} \rightarrow 0$.

[Geometry and applied fields in the magnetoconcentration effect (and also in the magnetodiode effect). Shape of transverse carrier profiles $n(y)$ and $I(V)$ characteristic for $s_{1} \rightarrow \infty$ and $s_{2} \rightarrow 0$.]

En régime permanent, les densités de courant dues aux électrons (en densité $n$ ) ou aux trous ( $p$ ) s'écrivent :

avec :

$$
\mathbf{i}_{n, p}=\left(\mathbf{i}_{n, p}\right)_{0}+\left(\mathbf{i}_{n, p}\right)_{0} \wedge \mu_{\mathrm{H}_{n, p}} \mathbf{B}
$$

$$
\left(\mathbf{i}_{n, p}\right)_{0}=\sigma_{n, p} \mathbf{E}-q_{n, p} D_{n, p} \nabla n, p
$$

où $\mu_{\mathrm{H}}$ est la mobilité (algébrique) de Hall, $\sigma$ la conduc- tivité, $D$ le coefficient de diffusion et ( $q$ étant la charge élémentaire) $q_{n}=-q, q_{p}=+q$.

On utilise la quasi-neutralité électrique $(\delta n \simeq \delta p)$ ainsi que les équations de continuité (conservation du nombre de porteurs) avec des lois de recombinaison qu'on supposera linéaires en volume comme en surface :

$$
\begin{aligned}
\operatorname{div} \mathbf{i}_{n, p} & =-q_{n, p} \frac{\delta n}{\tau_{\mathrm{v}}} \\
\left(\mathbf{i}_{n, p}\right)_{ \pm b / 2} & = \pm q_{n, p} s_{1,2} \delta n\left( \pm \frac{b}{2}\right)
\end{aligned}
$$

où $\tau_{\mathrm{v}}$ est la durée de vie volumique des porteurs et $s_{1,2}$ les vitesses de recombinaison sur les surfaces en $y= \pm \frac{b}{2}$.

On obtient alors l'équation différentielle qui régit la distribution transversale $\delta n(y)$ avec ses conditions aux limites. Pour un semiconducteur intrinsèque, l'équation différentielle peut s'écrire :

$$
\frac{\partial^{2} n}{\partial y^{2}}+\frac{1}{l_{\mathrm{c}}} \frac{\partial n}{\partial y}-\frac{1}{L_{\mathrm{a}}^{2}} \delta n=0
$$

où $L_{\mathrm{a}}$ est la longueur de diffusion ambipolaire des porteurs et $l_{\mathrm{c}}$ une longueur caractéristique des champs appliqués :

$$
l_{\mathrm{c}}=\frac{2 k T}{q}\left[\left(\left|\mu_{\mathrm{H} n}\right|+\mu_{\mathrm{H} p}\right) E_{x} B\right]^{-1} .
$$

La solution $n(y)$, qui peut s'exprimer analytiquement [3], confirme que le gradient de porteurs peut être très important, et produire une modification notable de la conductivité moyenne de dispositif [3-7].

Ainsi, un capteur à effet $\mathrm{MC}$ verra sa caractéristique courant-tension $I(V)$, ohmique en l'absence de champ magnétique (répartition uniforme des porteurs : $\left.n(y)=n_{0}\right)$, devenir fortement non linéaire lorsque $B \neq 0$. En particulier, dans le cas de surfaces « idéalement " dissymétriques $\left(s_{1} \rightarrow \infty\right.$ : recombinaison ou génération - de porteurs très intense en $y=+\frac{b}{2}$; $s_{2} \rightarrow 0:$ très peu de recombinaison en $y=-\frac{b}{2}$ ), la caractéristique $I(V)$ ressemble à une caractéristique de diode (Fig 1) :

- Si $E_{x} B>0$, les porteurs générés par la surface $\mathrm{s}_{1}$ sont accumulés près de la surface $s_{2}$; le semiconducteur étant ambipolaire, le déséquilibre peut devenir très important, sans qu'aucune charge d'espace n'apparaisse. Alors la conductivité moyenne augmente fortement, ainsi que le courant traversant le capteur.

- Si $E_{x} B<0$, les porteurs créés, en faible quantité, par $s_{2}$ sont déportés, sous l'action de la force de Laplace, vers la surface $s_{1}$ où ils se recombinent de façon intense, et le matériau se trouve globalement appauvri en porteurs. 
2.2 CAPTEURS À MAGNÉTOCONCENTRATION. - Il apparaît ainsi que la qualité des surfaces principales joue un rôle prépondérant dans la variation de résistance, donc dans la sensibilité magnétique, que manifestera un capteur basé sur l'effet MC. Le traitement de surface (du décapage chimique soigné, qui donne une faible recombinaison en surface, au polissage mécanique à l'abrasif, qui produit d'intenses recombinaisons) permettra donc de contrôler les performances d'un capteur à MC.

2.2.1 Sensibilité statique et sensibilité dynamique. Les simulations théoriques, utilisant les équations 1 à 6 , concordent parfaitement avec les résultats expérimentaux et montrent que les caractéristiques $\langle\sigma\rangle(B)$ ou $I(B)$, pour un champ $E_{x}$ donné (c'est-à-dire à tension $V$ constante), ont l'allure insérée en figure 2 (courbe en cloche).

$\mathrm{La}$ variation relative :

$$
\frac{I(B)}{I_{0}}=\frac{\langle i\rangle}{i_{0}}=\frac{\langle\sigma\rangle}{\sigma_{0}}=\frac{\langle\sigma\rangle}{\sigma_{n}+\sigma_{p}}
$$

vaut, pour un semiconducteur intrinsèque (même à très fort champ magnétique, comme nous l'avons montré récemment [7]) :

$$
\frac{I(B)}{I_{0}} \simeq \frac{\langle n\rangle}{n_{i}} .
$$

Elle correspond à une sensibilité statique $(\lessgtr 1)$, intéressante si $I(B) \gg I_{0}$, ou si $I(B) \rightarrow 0$.

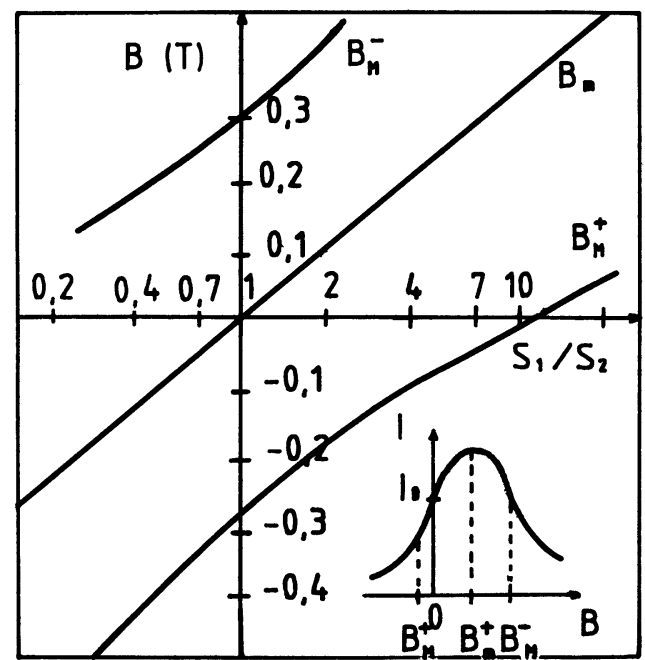

Fig. 2. - Valeurs des champs magnétiques conduisant au maximum de conduction $\left(B_{m}\right)$ et aux sensibilités dynamiques $S_{I}$ maximales $\left(B_{\mathrm{M}}^{+}\right.$et $\left.B_{\mathrm{M}}^{-}\right)$en fonction du rapport des recombinaisons en surface $s_{1} / s_{2}$. Capteur à $\mathrm{MC}$ en germanium intrinsèque : épaisseur $b=1 \mathrm{~mm} ; \tau_{\mathrm{v}}=100 \mu \mathrm{s} ; E_{x}=$ $10^{3} \mathrm{~V} / \mathrm{m} ; s_{1}=10 \mathrm{~m} / \mathrm{s} ;$ température ambiante.

[Magnetic fields corresponding to the maxima of conductivity $\left(B_{\mathrm{m}}\right)$ or of small-signal sensitivities $S_{I}\left(B_{\mathrm{M}}^{+}\right.$and $\left.B_{\mathrm{M}}^{-}\right)$ versus the ratio of surface recombination velocities $s_{1} / s_{2}$. MC sensor made from intrinsic germanium; room temperature.]
La sensibilité dynamique (en courant), $S_{I}=\frac{\Delta I}{\Delta B}=\frac{\partial I}{\partial B}$ est nulle pour le champ $B_{\mathrm{m}}$ correspondant au maximum de $I(B)$ et présente deux maxima pour des champs $B_{\mathrm{M}}^{+}$et $B_{\mathrm{M}}^{-}$situés de part et d'autre de $B_{\mathrm{m}}$ dont les valeurs sont déterminées par les recombinaisons en surface $s_{1}$ et $s_{2}$. La figure 2 montre, pour un capteur à $M C$ au germanium, l'évolution des champs $B_{m}, B_{M}^{+}$ et $B_{M}^{-}$en fonction du rapport $s_{1} / s_{2}$; on constate en particulier que $B_{\mathrm{m}} \propto \ln \left(s_{1} / s_{2}\right)$, qui reste vrai même à plus forts champs comme nous avons pu le montrer analytiquement [8]. De telles courbes permettent de choisir, selon le fonctionnement souhaité, les traitements de surface appropriés.

Signalons que, sans optimisation particulière, les capteurs à $\mathrm{MC}$ au germanium offrent à température ambiante des sensibilités moyennes de $100 \mathrm{~mA} / \mathrm{T}$ (à tension constante), ou $30 \mathrm{~V} / \mathrm{T}$ (à courant constant), déjà meilleures que celles de l'effet Hall et comparables à bas champ magnétique aux capteurs magnétorésistifs à InSb, avec l'avantage de détecter le sens du champ.

2.2.2 Optimisation. - Tant que les champs appliqués restent faibles, c'est-à-dire (cf. Eq. 6) $l_{\mathrm{c}} \gg L_{\mathrm{a}}$, il apparaît (Eq. 5) que la répartition transversale est essentiellement gouvernée par la diffusion et donc que, pour avoir une perturbation optimale de la conductivité, l'épaisseur $b$ du capteur doit être de l'ordre de la longueur de diffusion :

$$
b \sim L_{\mathrm{a}} .
$$

Cette relation a été longtemps considérée comme la seule condition d'optimisation relative aux dimensions des capteurs à MC. Nous avons montré et vérifié expérimentalement $[8,9]$ qu'en réalité dès que l'intensité des champs augmente et que $l_{\mathrm{c}}<L_{\mathrm{a}}, l_{\mathrm{c}}$ devient la longueur caractéristique du confinement ("pincement $")$ des porteurs près de la surface d'accumulation ; l'épaisseur optimale des capteurs sera alors $b$, telle que :

$$
L_{\mathrm{a}} \gg b \sim l_{\mathrm{c}} \text {. }
$$

Pour des surfaces fortement différentes $\left(s_{1} \gg s_{2}\right)$ et des champs appliqués conduisant à une accumulation près de $s_{2}$, et tels que $l_{\mathrm{c}} \ll L_{\mathrm{a}}, L_{\mathrm{a}}^{2} / b$, la résolution de l'équation (5) montre que l'épaisseur optimale correspond à $: \exp \left(b / l_{\mathrm{c}}\right) \simeq\left(s_{1} / s_{2}\right)\left(b / l_{\mathrm{c}}-1\right)$, soit :

$$
b \simeq l_{\mathrm{c}} \ln \left(s_{1} / s_{2}\right)
$$

mais surtout, il apparaît que, dans ces conditions, on obtient une sensibilité statique nettement supérieure (augmentée de plus d'un ordre de grandeur), et valant :

$$
\frac{I(B)}{I_{0}}=\left(\frac{\operatorname{sh}\left(b / 2 l_{c}\right)}{b / 2 l_{c}}\right)^{2} \simeq \frac{s_{1} / s_{2}}{\ln \left(s_{1} / s_{2}\right)} .
$$

De plus, on peut montrer [10] que la relation 10 correspond aussi à peu près à une sensibilité dynamique optimale pour des fluctuations d'un champ magnétique autour d'une valeur moyenne $B \neq 0$. 


\section{L'effet magnétodiode.}

Malgré leurs bonnes performances, le développement des capteurs à $\mathrm{MC}$ est limité par le fait que l'effet physique sur lequel ils sont basés n'est important que dans les semiconducteurs ambipolaires (et pour des applications pratiques, essentiellement le $\mathrm{Ge}$ et l'InSb), donc en général sensible à la température, mais aussi non utilisables pour les techniques d'intégration actuelles.

Les potentialités de l'effet MC ont pu être utilisées dans les semiconducteurs extrinsèques et dans les matériaux de la micro-électronique en recréant un comportement bipolaire par injection de porteurs par les contacts électriques.

L'effet magnétodiode (MD) peut donc être décrit simplement comme un effet de magnétoconcentration sur les porteurs injectés par la ou les diode(s) d'extrémité.

Les MD à un seul contact injectant (diodes « longues » du type $p^{+} n$ ou $p^{+}$i) $[11,12]$ sont essentiellement gouvernées par des phénomènes de diffusion dans la "base » (partie n ou i) du dispositif. Leurs performances comme capteurs magnétiques sont en général inférieures à celles des structures à deux contacts injectants [13]. Nous ne discuterons donc ici que des MDà deux contacts injectants, du type $\mathrm{p}^{+} \mathrm{nn}^{+}$ ou $\mathrm{p}^{+}$in $^{+}$.

3. 1 BASES THÉORIQUES. - L'échantillon semiconducteur (ou «base», de dopage $n_{0}$ et de longueur $L$ ) est muni d'un contact injectant des trous (jonction $\mathrm{p}^{+} \mathrm{n}$ ) et d'un contact injectant des électrons (jonctions $\mathrm{nn}^{+}$). La configuration des champs appliqués reste celle de la figure 1. En l'absence de champ magnétique, les caractéristiques courant-tension d'une telle structure correspondent aux divers régimes de double injection dans un semiconducteur (voir [14]) et sont non linéaires dès que le niveau d'injection est suffisant, ce qui est nécessaire dans notre cas pour que l'effet MC soit efficace.

En présence d'un champ magnétique, la nonuniformité longitudinale de la répartition des porteurs propre à la double injection va se doubler d'un déséquilibre transversal provoqué par l'effet MC. En combinant les équations 1 à 3 et l'équation de Poisson, et conservant les termes prépondérants, l'équation qui régit la distribution de porteurs $n(x, y)$ s'écrit alors :

$$
\frac{\partial^{2} n}{\partial y^{2}}+\frac{1}{l_{\mathrm{c}}} \frac{\partial n}{\partial y}-\frac{\delta n}{L_{\mathrm{a}}^{2}}=-\frac{G(x)}{D_{\mathrm{a}}}
$$

où $D_{\mathrm{a}}$ est le coefficient de diffusion ambipolaire et $G(x)$, analogue à un taux de création de porteurs, est tel que :

$$
\frac{G(x)}{D_{\mathrm{a}}}=\frac{q}{2 k T} n_{0} \frac{\mathrm{d} E_{x}}{\mathrm{~d} x}-\frac{1}{2 k T} \varepsilon \frac{\mathrm{d}}{\mathrm{d} x}\left(E_{x} \frac{\mathrm{d} E_{x}}{\mathrm{~d} x}\right)+\frac{\partial^{2} n}{\partial x^{2}} .
$$

Le terme de gauche de l'équation 12 est analogue à celui de l'équation 5, propre à l'effet $\mathrm{MC}$. La solution de l'équation 12 avec ses conditions aux limites (4) peut se mettre sous la forme $[15,13]$ :

$$
n(x, y) \simeq \delta n(x, y)=\delta n_{\mathrm{c}}(y)+\tau_{\mathrm{v}} G(x)(1+g(y))
$$

où $\delta n_{\mathrm{c}}(y)$ correspond à la répartition des porteurs qui résulterait du seul effet MC.

On obtient ensuite, par intégration, la densité de courant moyenne $i$ traversant la structure en fonction de la tension appliquée

$$
V=-\int_{0}^{L} E_{x}(x) \mathrm{d} x
$$

Selon le terme prépondérant dans l'équation 13 , on peut retrouver alors [13] des relations couranttension correspondant formellement aux divers régimes de la double injection :

— régime " semiconducteur » :

$$
i(V, B)=\left(\frac{9}{8}\right) q\left|\mu_{n}\right| \mu_{p} n_{0} \tau_{\mathrm{sc}} V^{2} / L^{3}
$$

— régime « isolant » :

$$
i(V, B)=\left(\frac{125}{18}\right) \varepsilon\left|\mu_{n}\right| \mu_{p} \tau_{\text {is }} V^{3} / L^{5}
$$

— puis les régimes "de diffusion ".

Les paramètres $\tau_{\text {sc }}$ et $\tau_{\text {is }}$ peuvent être interprétés comme des durées de vie "effective" des porteurs (voir [4]); il est à remarquer qu'elles dépendent non seulement des recombinaisons en volume $\left(\tau_{v}\right)$, en surface $\left(s_{1}, s_{2}\right)$ et de l'épaisseur $b$, mais aussi des champs appliqués $(B ; V / L), \ldots$ et que l'allure des caractéristiques (quadratique, cubique, ...) est donc susceptible d'être modifiée par l'influence d'un champ magnétique [13]. Celle-ci est résumée sur la figure 3 qui traduit schématiquement les sensibilités au champ magnétique d'une MD.

Remarquons que la sensibilité dynamique est telle que :

$$
S_{I} \propto \frac{\partial I}{\partial B} \propto \frac{\partial \tau}{\partial B},
$$

alors qu'une sensibilité dynamique relative est donnée par :

$$
S_{I}^{*}=\frac{\partial I}{I \partial B}=\frac{\partial i}{i \partial B}=\frac{\partial \tau}{\tau \partial B} .
$$

L'optimisation du fonctionnement des MD comme capteurs dépend ainsi :

- de facteurs géométriques (essentiellement sa longueur $L$ ) afin qu'un niveau d'injection suffisant soit atteint dans le dispositif;

- du traitement de surfaces, selon le type de sensibilité recherchée (voir § 2.2.1); 


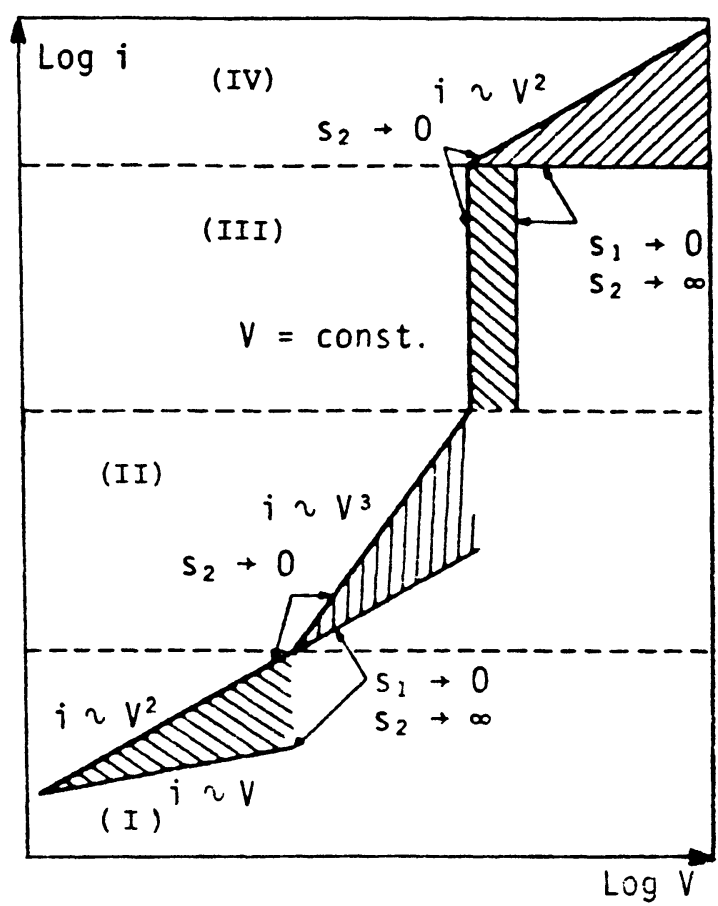

Fig. 3. - Influence d'un champ magnétique $B>0$ (porteurs déportés vers la surface $s_{2}$ ) sur les caractéristiques $I(V)$ des magnétodiodes $\mathrm{p}^{+} \mathrm{nn}^{+}$selon les divers régimes de double injection ((I) : régime semiconducteur; (II) : régime isolant ; (III) : régime de diffusion, à niveau modéré ; (IV) : diffusion, à très fort niveau). Remarquons que les évolutions extrêmes correspondent à $s_{2} \rightarrow 0$ et $s_{2} \rightarrow \infty$.

[Influence of a magnetic field $B>0$ (carriers deflected towards $s_{2}$ surface) on the $I(V)$ characteristics of $\mathrm{p}^{+} \mathrm{nn}^{+}$ magnetodiodes, according to the double injection regime ((I) : semiconductor regime; (II) : insulator regime; (III) : diffusion regime at moderate level; (IV) : diffusion, very high level). Note that the extreme evolutions correspond to $s_{2} \rightarrow 0$ and $s_{2} \rightarrow \infty$.]

- de paramètres relatifs à l'intensité des champs (appliqués ou à détecter) par rapport à l'épaisseur b du dispositif. A ce sujet, nous venons de montrer [10] qu'en ce qui concerne la sensibilité statique et la sensibilité dynamique relative $\left(S_{I}^{*}\right)$, les conclusions énoncées pour les capteurs à $\mathrm{MC}(\S 2.2 .2)$ s'appliquent presque telles quelles aux MD.

\subsection{RÉALISATIONS EXPÉRIMENTALES.}

3.2.1 Magnétodiodes au germanium. - Le germanium a été le matériau semiconducteur idéal pour l'expérimentation des premières MD [11, 23-25], et permet encore d'obtenir les capteurs les plus sensibles $[13,16]$. Son inconvénient principal pour nos applications est donc de ne pas pouvoir permettre la réalisation de microcapteurs intégrés. A partir d'échantillons de type $\mathrm{n}$ peu dopés (résistivités 30 à $50 \Omega . \mathrm{cm}$ ) de section $\sim \mathrm{mm}^{2}$ et de longueur $L=2$ à $10 \mathrm{~mm}$, nous avons réalisé par diffusion d'indium (ou d'étain) les jonctions $\mathrm{p}^{+} \mathrm{n}\left(\right.$ ou $\mathrm{nn}^{+}$). L'influence des principaux paramètres $(V, B$, mais aussi recombinaisons en surface) $[13,16]$ est en très bon accord avec l'analyse théorique, et des sensibilités dynamiques élevées ont été atteintes, par exemple :

$$
\Delta V / \Delta B \sim 300 \mathrm{~V} / \mathrm{T} ; \quad \Delta \mathrm{I} / \Delta B \sim 30 \mathrm{~A} / \mathrm{T} .
$$

3.2.2 Micromagnétodiodes intégrées (silicium sur corindon). - a) Magnétodiodes SOS. Nous avons étudié, pour optimisation, des micromagnétodiodes fabriquées par le LETI-CENG selon la technologie S.O.S. ("Silicon On Sapphire "). Cette technologie, à cause même de ses défauts notamment à l'interface $\mathrm{Si}-\mathrm{Al}_{2} \mathrm{O}_{3}$ [17] est a priori un bon matériau pour la réalisation de MD : l'épaisseur standard des couches de Si épitaxié $(b=0,65 \mu \mathrm{m})$ est légèrement inférieure à la longueur de diffusion des porteurs et les deux interfaces $\left(\mathrm{Si}_{-} \mathrm{SiO}_{2}\right.$ et $\mathrm{Si}-\mathrm{Al}_{2} \mathrm{O}_{3}$ ) présentent macroscopiquement des taux de recombinaison très différents ce qui, d'après la figure 3 , doit conduire à une sensibilité optimale.

Puisqu'on ne peut modifier efficacement ni l'épaisseur $b$, ni la qualité des surfaces, les paramètres permettant une optimisation [18] sont le dopage initial $n_{0}$ ainsi que la longueur $L$ et la largeur $Z$ des dispositifs. Il apparaît qu'afin de permettre une injection suffisante, le dopage $n_{0}$ doit rester faible $\left(n_{0}<10^{23} \mathrm{~m}^{-3}\right)$ et la MD doit être courte $(L<30 \mu \mathrm{m})$, ce qui évite aussi de travailler à des tensions trop élevées. La largeur $Z$ détermine uniquement le courant total traversant la structure.

Les tableaux I et II montrent quelques valeurs des sensibilités obtenues (à tension ou à puissance constante) à température ambiante.

Tableau I. - Sensibilités magnétiques absolues $S_{\mathrm{I}}=\Delta I / \Delta B(\mathrm{~mA} / \mathrm{T})$ et relatives $S_{\mathrm{I}}^{*}=\Delta I / I \Delta B\left(10^{-2} / \mathrm{T}\right)$ de micromagnétodiodes S.O.S. de dopage $\mathrm{n}_{0}$ et de longueur $L$, pour une tension $V=10 \mathrm{~V}$.

[Magnetic sensitivities, absolute $S_{I}$ or relative $S_{I}^{*}$, for S.O.S. micromagnetodiodes with length $L$ and doping $\mathrm{n}_{0}$, at $V=10 \mathrm{~V}$.]

\begin{tabular}{|c|c|c|c|c|c|c|}
\hline \multirow{2}{*}{$\begin{array}{c}\text { dopage } \begin{array}{c}\text { do } \\
\left(\mathrm{m}^{-3}\right)\end{array} \\
\text { longueur } L\end{array}$} & \multicolumn{2}{|c|}{$5 \times 10^{21}$} & \multicolumn{2}{|c|}{$7 \times 10^{21}$} & \multicolumn{2}{|c|}{$2 \times 10^{22}$} \\
\cline { 2 - 7 }$(\mu \mathrm{m})$ & $S_{I}$ & $S_{I}^{*}$ & $S_{I}$ & $S_{I}^{*}$ & $S_{I}$ & $S_{I}^{*}$ \\
\hline 10 & 2,2 & 21 & 2,1 & 20 & 1 & 12 \\
20 & 0,5 & 20 & 2 & 18 & 0,8 & 10 \\
30 & 0,1 & 10 & 0,2 & 12 & 0,2 & 7 \\
50 & 0,05 & 8 & 0,1 & 9 & 0,1 & 5 \\
\hline
\end{tabular}

b) Magnétodiodes SOS à contact Schottky. La MD à contact Schottky $[19,20]$ a été inventée dans notre laboratoire (Brevet 1977) : il s'agit d'un tripôle combinant une $\mathrm{MD}\left(\mathrm{p}^{+} \mathrm{nn}^{+}\right)$polarisée en direct et une diode métal-semiconducteur (contact Schottky) montée sur la base $n$ de la MD et polarisée en inverse (Fig. 4). Sa sensibilité provient de la modulation du 
Tableau II. - Sensibilités magnétiques absolues $S_{I}=\Delta I / \Delta B \quad(\mathrm{~mA} / \mathrm{T}), \quad S_{V}=\Delta V / \Delta B(V / T)$ et relatives $S_{I}^{*}=\Delta I / I \Delta B\left(10^{-2} / \mathrm{T}\right), S_{V}^{*}=\Delta V / V \Delta B\left(10^{-2} / \mathrm{T}\right)$ de micromagnétodiodes S.O.S. de dopage $n_{0}$ et de longueur $L$, pour une puissance dissipée de 40 à $60 \mathrm{~mW}$.

[Magnetic sensitivities, absolute $S_{I}, S_{V}$ or relative $S_{I}^{*}, S_{V}^{*}$ for S.O.S. micromagnetodiodes with length $L$ and doping $n_{0}$, for a power dissipation 40 to $60 \mathrm{~mW}$.]

\begin{tabular}{|c|c|c|c|c|c|c|c|c|c|c|c|c|c|c|c|c|}
\hline \multirow{2}{*}{$\mid \begin{aligned} & \quad \quad \begin{array}{r}\text { dopage } \mathrm{n}_{0} \\
\left(\mathrm{~m}^{-3}\right)\end{array} \\
& \text { longueur } \\
& L(\mu \mathrm{m})\end{aligned}$} & \multicolumn{4}{|c|}{$5 \times 10^{21}$} & \multicolumn{4}{|c|}{$7 \times 10^{21}$} & \multicolumn{4}{|c|}{$2 \times 10^{22}$} & \multicolumn{4}{|c|}{$2 \times 10^{23}$} \\
\hline & $S_{I}$ & $S_{I}^{*}$ & $S_{V}$ & $S_{V}^{*}$ & $S_{I}$ & $S_{I}^{*}$ & $S_{V}$ & $S_{V}^{*}$ & $S_{I}$ & $S_{I}^{*}$ & $S_{V}$ & $S_{V}^{*}$ & $S_{I}$ & $S_{I}^{*}$ & $S_{V}$ & $S_{V}^{*}$ \\
\hline 10 & 1,12 & 18 & 1,4 & 14 & 2,2 & 21 & 0,75 & 13 & 0,75 & 16 & 0,9 & 12 & 0,3 & 1 & 0,02 & 1 \\
\hline 20 & 0,28 & 15 & 3 & 12 & 0,32 & 12 & 1 & 10 & 0,65 & 10 & 1 & 10 & 0,2 & 1 & 0,05 & 1 \\
\hline 30 & 0,22 & 15 & 6 & 14 & 0,25 & 15 & 5 & 17 & 0,5 & 10 & 4 & 15 & 0,16 & 1 & 0,06 & 1 \\
\hline 50 & 0,2 & 22 & 13 & 18 & 0,125 & 13 & 7 & 13 & 0,4 & 10 & 7 & 20 & 0,13 & 1 & 0,08 & 1 \\
\hline
\end{tabular}

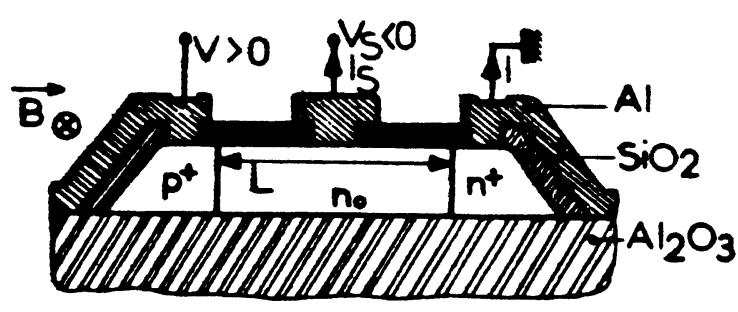

Fig. 4. - Configuration et polarisations d'une micromagnétodiode SOS à contact Schottky.

[Outline and bias of a SOS Schottky magnetodiode.]

courant inverse de la diode Schottky par la densité de porteurs $n_{\mathrm{s}}$ présents à la limite de la zone de charge d'espace et de la partie neutre de la base; cette densité varie lorsqu'on applique un champ magnétique par suite de l'effet MD (et de la concentration de porteurs par le champ magnétique près de la surface à faible recombinaison). Si le courant de saturation $I_{S}$ de la diode Schottky est dû essentiellement au terme de diffusion, nous avons alors :

$$
\delta I_{\mathrm{S}} / I_{\mathrm{s}}=\delta n_{\mathrm{s}}\left(-\frac{b}{2}\right) / n_{\mathrm{s}} .
$$

Les MD à contact Schottky présentent une très bonne sensibilité en tension (Fig. 5); cette sensibilité est typiquement 3 à 15 fois meilleure que celle de la MD d'origine, à même niveau d'injection, car la densité de porteurs à l'interface $\mathrm{Si}_{-} \mathrm{SiO}_{2}$ est plus sensible au champ magnétique que la densité moyenne des porteurs.

\section{Autres performances et limitations.}

4. 1 Tenue en Température. - Les capteurs $a ̀$ MC sont, par nature (ils utilisent des semiconducteurs proches de l'état intrinsèque) très sensibles aux varia-
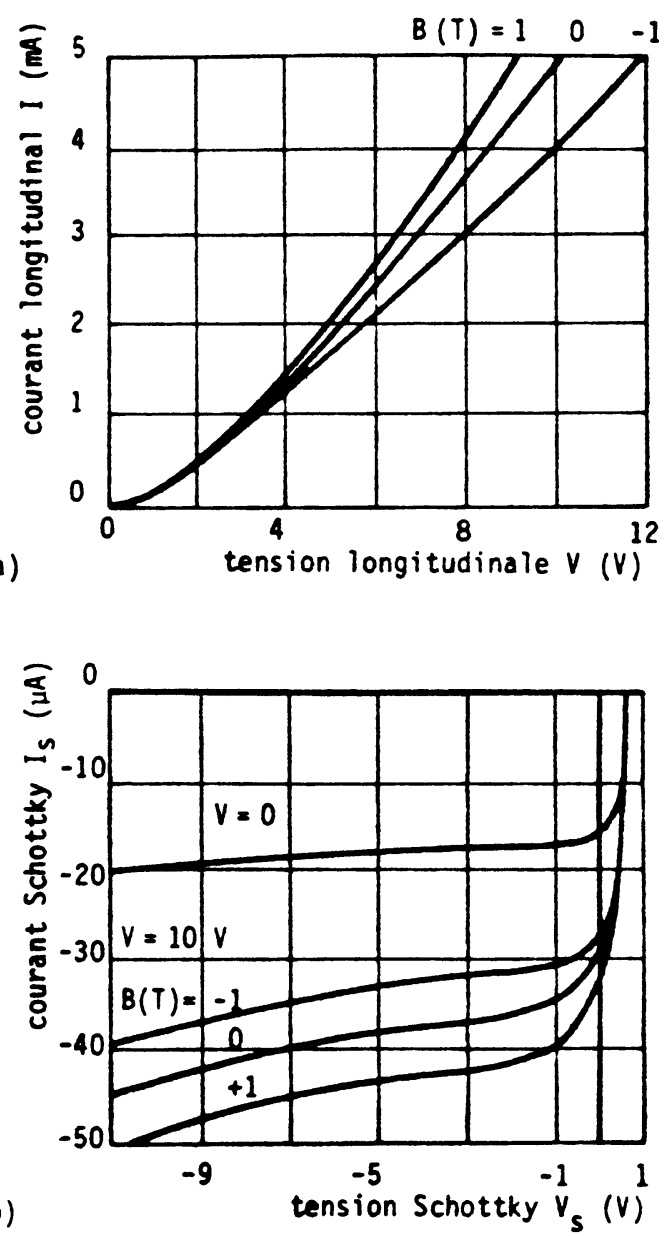

Fig. 5. - Influence d'un champ magnétique $(a)$ sur la caractéristique $I(V)\left(\mathrm{a} I_{\mathrm{S}}=0\right)$ et $(b)$ sur la caractéristique inverse $I_{\mathbf{S}}\left(V_{\mathrm{S}}\right)$ du contact Schottky dans une micromagnétodiode S.O.S. $\left(n_{0}=5 \times 10^{21} \mathrm{~m}^{-3} ; b=0.65 \mu \mathrm{m}\right.$; $L=20 \mu \mathrm{m} ; Z=200 \mu \mathrm{m} ;$ contact Schottky : $6 \times 40 \mu \mathrm{m}^{2}$; température ambiante).

[Influence of a magnetic field, at room temperature, $(a)$ on the $I(V)$ characteristic (with $I_{\mathrm{S}}=0$ ) and $(b)$ on the reverse characteristic $I_{\mathbf{S}}\left(V_{\mathrm{S}}\right)$ of the Schottky contact in a S.O.S. micromagnetodiode.] 
tions de température : leurs sensibilités absolues au champ magnétique $S_{\mathrm{I}}$ ou $S_{\mathrm{V}}$ varient fortement avec la température. Par contre, leurs sensibilités relatives $\left(S_{\mathrm{I}}^{*}\right.$ ou $\left.S_{\mathrm{V}}^{*}\right)$ sont pratiquement indépendantes de la température. Signalons aussi que les impuretés résiduelles du matériau rendront les capteurs au germanium inopérants en dessous de $-30^{\circ}$ à $-50^{\circ} \mathrm{C}$. Par contre, les capteurs à InSb peuvent fonctionner sans difficultés jusqu'à $-200^{\circ} \mathrm{C}$.

Dans les magnétodiodes, la température intervient de deux façons :

- la température peut gouverner l'existence même des effets d'injection (et donc de l'effet MD) : c'est le cas des MD intégrées en Silicium Sur Saphir où, si entre $-120^{\circ} \mathrm{C}$ et $+100^{\circ} \mathrm{C}$ les caractéristiques des diodes courtes $(L=10 \mu \mathrm{m}$ ou $20 \mu \mathrm{m})$ ne sont pas trop modifiées, le régime de double injection n'apparaît plus à basse température pour les diodes longues $(L=50 \mu \mathrm{m})$;

- la température peut modifier les performances d'une MD : ainsi, pour les MD en Ge, comme pour celles en S.O.S., la sensibilité absolue en tension $S_{\mathrm{v}}$ augmente lorsque la température diminue (typiquement multipliée par 3 à 5 entre la température ambiante et $-100^{\circ} \mathrm{C}$ ) alors que la sensibilité relative $S_{v}^{*}$ varie peu. Par contre, la sensibilité en courant diminue assez nettement à basse température.

4.2 BRUIT. - Le bruit propre d'un capteur détermine sa sensibilité ultime (c'est-à-dire sa limite de détection).

Dans les capteurs à $M C$, le bruit est assez faible : par exemple, pour des capteurs au germanium, sa densité spectrale de puissance est inférieure, à $1 \mathrm{kHz}$, à $10^{-14} \mathrm{~V}^{2} / \mathrm{Hz}$; son spectre est en $1 / f$ en dessous de $1 \mathrm{kHz}$ et fait apparaître des bruits de générationrecombinaison et de diffusion au-delà. Mais ce bruit est très sensible au champ magnétique; nous avons montré [21] qu'un bruit en $1 / f$ dû à des effets de surface peut multiplier par $10^{3}$ la densité spectrale des fluctuations basse fréquence pour un champ de $0,5 \mathrm{~T}$.

Dans les magnétodiodes, le bruit est plus élevé (au moins un ordre de grandeur dans des conditions comparables). Il est en $1 / f$ jusqu'à environ $10 \mathrm{kHz}$ pour les deux types de MD étudiés (Ge et S.O.S.). Nous avons pu montrer récemment [22] que ce bruit provient de fluctuations de conductivité dans la base (qui constituent aussi la source de bruit prépondérante dans une MD à contact Schottky); la densité spectrale des fluctuations de tension recueillies aux bornes de la $\mathrm{MD}$, lorsque celle-ci travaille à un niveau d'injection suffisant dans le régime semiconducteur, est ainsi proportionnelle à :

$$
L i^{2} / Z b \delta n^{3}(L) f \propto L V / Z b n_{0} f .
$$

Cette relation rend parfaitement compte des variations du bruit mesuré avec le dopage initial $n_{0}$, la polarisation $(I, V)$ et les dimensions de la $\operatorname{MD}(L, Z, b)$, confirmant l'avantage des structures courtes $(L \leqslant 20 \mu \mathrm{m})$. Elle permet aussi d'expliquer l'évolution du bruit :

- avec le champ magnétique appliqué : le bruit varie dans le même sens que la résistance de la MD;

- avec la température : il augmente quand la température diminue (car la résistance de la MD augmente); par exemple, pour une $\mathrm{MD}$ en $\mathrm{Ge}$, la densité spectrale du bruit est typiquement multipliée par 15 (à courant constant) lorsque la température passe de $+20^{\circ} \mathrm{C}$ à $-50^{\circ} \mathrm{C}$ (alors que la résistance augmente d'un facteur 2,5). Mais comme, dans le même temps, la sensibilité magnétique correspondante est multipliée par 4, il apparaît que la limite de détection des MD est très peu affectée par la température.

\section{Conclusion.}

Les effets basés sur la concentration de porteurs par un champ magnétique (magnétoconcentration ou magnétodiode) conduisent à des capteurs magnétiques à semiconducteur présentant d'excellentes performances. De conception et d'utilisation simple (en règle générale ce sont des dipôles, à l'exception du tripôle que constitue la magnétodiode à contact Schottky), ces capteurs ont des sensibilités « ajustables " par contrôle des recombinaisons en surface, et pour la plupart meilleures que celles des autres capteurs à semiconducteur (effet Hall ou magnétorésistance).

Mais surtout, les magnétodiodes peuvent maintenant être intégrées grâce aux technologies de couches minces de Silicium Sur Isolant, ouvrant peut-être une voie vers la réalisation de capteurs intelligents.

\section{Remerciements.}

Nous tenons à remercier la D.G.R.S.T. et le G.I.S. de Mini-Micro-Informatique qui ont financé une partie de ces recherches, ainsi que le LETI-C.E.N. Grenoble qui a fabriqué les prototypes de micromagnétodiodes S.O.S.

\section{Bibliographie}

[1] WeIss, H., Magnetoresistance, chap. 10 in Semiconductors and Semimetals, Vol. 1 : Physics of III-V compounds, Willardson, R. K. et Beer, A. C. Ed. (Academic Press, New-York) 1966.
[2] WEISs, H., Structure and application of galvanomagnetic devices (Pergamon Press, Oxford) 1969.

[3] Madelung, O., Tewordt, L. et Welker, H., $Z$. Naturforschg 10a (1955) 476-488. 
[4] Chovet, A. et Kamarinos, G., Revue Phys. Appl. 6 (1971) 345-354.

[5] Chovet, A., Phys. Status Solidi (a) 28 (1975) 633-645.

[6] Cristoloveanu, S. et Lee, J. H., J. Phys. C 13 (1980) 5983-5997.

[7] Cristoloveanu, S., Chovet, A. et Lakeou, S., J. Phys. C 16 (1983) 927-938.

[8] Chovet, A., Cristoloveanu, S., Daher, Y. et MalyuTENKo, V. K., Phys. Status Solidi (a), 76 (1983) K43-K48.

[9] Malyutenko, V. K., MalosovskiI, Yu. M., SAShChuk, A. P. et Chovet, A., Phys. Status Solidi (a) 71 (1982) 217-222.

[10] Cristoloveanu, S., Chovet, A. et Malyutenko, V. K., Proc. 2nd Int. Conf. on Solid State Sensors and Actuators Solid State Transducers 83, Delft, Netherlands (may 31-june 3, 1983) Sensors Actuators 4 (1983) 165-171.

[11] Stafeev, V. I., Sov. Phys. Solid State 1 (1959) 763-768.

[12] Karakushan, E. I. et Stafeev, V. I., Sov. Phys. Semicond. 9 (1975) 953-955.

[13] Cristoloveanu, S., Phys. Status Solidi (a) 64 (1981) 683-695 et 65 (1981) 281-292.

[14] Baron, R. et MaYer, J. W., Double injection in semiconductors, chap. 4 in Semiconductors and Semimetals, Vol. 6 : Injection phenomena, Willardson, R. K. et Beer, A. C. Ed. (Academic Press, New-York) 1970.

[15] Pfleiderer, H., Solid State Electron. 15 (1972) 335-353.

[16] Cristoloveanu, S., Onde Elect. 59 (1979) no 5 68-74.

[17] Cristoloveanu, S., Chovet, A. et Kamarinos, G., Revue Phys. Appl. 13 (1978) 615-618.

[18] Mohaghegh, A., Cristoloveanu, S. et De PontCHARRA, J., IEEE Trans. Electron Devices ED 28 (1981) 237-242.

[19] Chrétien, J., Kamarinos, G. et Viktorovitch, P., Revue Phys. Appl. 12 (1977) 1699-1703.

[20] Cristoloveanu, S., Mohaghegh, A. et DE PontCharra, J., J. Physique Lett. 41 (1980) L-235L-237.
[21] Dilmi, T., Chovet, A. et Viktorovitch, P., J. Appl. Phys. 50 (1979) 5348-5351.

[22] Chovet, A., Cristoloveanu, S., Mohaghegh, A. et Dandache, A., Proc. 2nd Int. Conf. on Solid State Sensors and Actuators Solid State Transducers 83, Delft, Netherlands (may 31-june 3, 1983), Sensors Actuators 4 (1983) 147-153.

[23] Stafeev, V. I. et Karakushan, E. I., Magnétodiodes (en russe), Nauka, Moscou (1975).

[24] Gribnikov, Z. S., Lomova, G. I. et Romanov, V. A., Phys. Status Solidi 28 (1968) 815-825.

[25] Yamada, T., Proc. 9th Int. Conf. on Physics of Semiconductors, Moscou (1968) vol. 2, 672-675.

[26] Lilienkamp, P. et Pfleiderer, H., Phys. Status Solidi (a) 43 (1977) 479-486.

[27] Lutes, O. S., Nussbaum, P. S. et Aadland, O. S., IEEE Trans. Electron Devices ED 27 (1980) 2156-2157.

[28] Popovic, R. S. et Baltes, H. P., 12th Eur. Sol. State Device Res. Conf. ESSDERC. 82, Munich (1982), Abstracts, 96-97.

[29] Lysenko, V. S., LitovskiI, R. N., Roumenin, Ch. S. et Smirnov, N. D., Revue Phys. Appl. 18 (1983) 87-92.

[30] Popovic, R. S. et Baltes, H. P., A CMOS magnetic field sensor (en cours de publication).

[31] Vikulin, I. M., Kanishcheva, N. A., Glauberman, M. A. et Vikulina, L. F., Sov. Phys. Semicond. 9 (1976) 1011-1013.

[32] Kozlov, O. M., Rekalova, G. I., Mitnikova, I. M., Tairova, D. A. et Shakhov, A. A., Sov. Phys. Semicond. 13 (1979) 1263-1265.

[33] Zieren, V. et Duyndam, B. P. M., IEEE Trans. Electron Devices ED 29 (1982) 83-90.

[34] Vinal, A. W., IBM J. Res. Develop. 25 (1981) 196-201.

[35] Lopez, J. L. et Licini, J. C., J. Appl. Phys. 53 (1982) 8389-8391.

[36] Brini, J. et Kamarinos, G., Sensors and Actuators 2 (1981/82) 149-154. 